\title{
Low-drag events in transitional wall-bounded turbulence
}

\author{
Richard D. Whalley, ${ }^{1,2}$ Jae Sung Park, ${ }^{3,4}$ Anubhav Kushwaha, ${ }^{3}$ David J. C. Dennis, ${ }^{2}$ \\ Michael D. Graham, ${ }^{3}$ and Robert J. Poole ${ }^{2, *}$ \\ ${ }^{1}$ School of Mechanical and Systems Engineering, Newcastle University, Newcastle NE1 7RU, United Kingdom \\ ${ }^{2}$ School of Engineering, University of Liverpool, Liverpool L69 3GH, United Kingdom \\ ${ }^{3}$ Department of Chemical and Biological Engineering, University of Wisconsin-Madison, \\ Madison, Wisconsin 53706, USA \\ ${ }^{4}$ Department of Mechanical and Material Engineering, University of Nebraska-Lincoln, \\ Lincoln, Nebraska 68588, USA
}

(Received 26 January 2016; published 6 March 2017)

\begin{abstract}
Intermittency of low-drag pointwise wall shear stress measurements within Newtonian turbulent channel flow at transitional Reynolds numbers (friction Reynolds numbers $70-130$ ) is characterized using experiments and simulations. Conditional mean velocity profiles during low-drag events closely approach that of a recently discovered nonlinear traveling wave solution; both profiles are near the so-called maximum drag reduction profile, a general feature of turbulent flow of liquids containing polymer additives (despite the fact that all results presented are for Newtonian fluids only). Similarities between temporal intermittency in small domains and spatiotemporal intermittency in large domains is thereby found.
\end{abstract}

DOI: 10.1103/PhysRevFluids.2.034602

\section{INTRODUCTION}

In the near-wall region of wall-bounded shear flows, particularly the buffer layer, turbulent flows are known to be dominated by coherent structures [1] comprised of quasistreamwise vortices staggered in the flow direction. Indeed, these near-wall structures export turbulent kinetic energy to the rest of the flow [2]. A number of related qualitative arguments have been put forward to explain the origin of this self-sustaining near-wall structure [2-5].

The understanding of shear flow turbulence at transitional Reynolds numbers has been greatly advanced by recent applications of dynamical systems theory to turbulent flow [6]. In particular, the discovery of three-dimensional fully nonlinear traveling wave (TW) solutions to the Navier-Stokes equations has enabled a priori study of self-sustained near-wall coherent structures that resemble in many ways transient structures observed in fully turbulent flows [7]. These solutions, also denoted as exact coherent states (ECS) [8], are steady states in a reference frame translating at a constant streamwise speed. They have been found numerically in all canonical wall-bounded geometries for turbulent flows [8-21]. These solutions arise in pairs at a saddle-node bifurcation point at a particular Reynolds number. The upper-branch solution of each pair has larger velocity fluctuations and higher drag compared to the lower-branch solution. They have a spatial structure of low-speed streaks that are wavy in the streamwise direction, straddled by streamwise vortices, and thus resemble the recurrent coherent structures in near-wall turbulence. As traveling waves these solutions are spatially periodic in the streamwise and spanwise flow directions and the spatial wavelengths at which they first appear are (1) close to observed correlation lengths in near-wall turbulence and (2) consistent with the smallest domain sizes (minimal channels) in which turbulence is found in direct numerical simulations (DNS) [3,22].

*robpoole@liverpool.ac.uk

Published by the American Physical Society under the terms of the Creative Commons Attribution 4.0 International license. Further distribution of this work must maintain attribution to the author(s) and the published article's title, journal citation, and DOI. 
Very recently, an ECS family with particularly intriguing behavior has been discovered in the plane channel flow geometry [23]. The mean velocity profile of the upper branch solution of the solution family denoted P4 in Ref. [23] closely approaches the classical (von Kármán) profile, while the lower branch velocity profile approaches a form generally associated with turbulence in viscoelastic polymer solutions, the so-called Virk profile [24]. This profile is a good approximation of experimental and computational observations in the so-called maximum drag reduction (MDR) regime and is insensitive to Reynolds number or polymer properties. It is the most general aspect of the drag reduction phenomenon. Other properties such as Reynolds shear stress level are not as general [25]. This ECS result adds to the set of observations in which mean velocity profiles close to the Virk profile are found in Newtonian flow [26-32], something that we also observe here. Notwithstanding this intriguing similarity to MDR, we emphasize that all results reported in the present work are for Newtonian fluid flows.

Furthermore, turbulent trajectories in the minimal geometry spend most of their time near the upper-branch solution, with occasional excursions toward the lower-branch solution [23]; similar observations have been made in the Couette geometry [16]. This result clarifies earlier observations $[28,29,32,33]$ of active and hibernating phases of minimal channel turbulence, showing that these correspond to time intervals where the trajectory is close to upper- and lower-branch traveling waves, respectively. Returning briefly to possible connections to viscoelastic turbulence we note that at low to moderate drag reduction, the hibernating phases increase in frequency while remaining similar in structure as the degree of viscoelasticity increases [32,34].

In this paper we present experimental observations of spatiotemporally local hibernating turbulence intervals in low-Reynolds-number Newtonian turbulent channel flow, the latter of which are characterized using conditional sampling techniques. These experimental findings are complemented by direct numerical simulation (DNS) results and comparisons with the recently discovered P4 family of ECS solutions discussed above [23]. The similarities between them observed here suggest that there is a significant connection, as yet not fully understood, between temporal intermittency in minimal domains and spatiotemporal intermittency in large domains. Together these results corroborate the dynamical picture of active and hibernating intervals, providing a connection between classical wall-bounded turbulence and ECS. Furthermore, both the low-drag hibernation intervals and the ECS solutions have a mean velocity profile close to that observed at MDR, thus pointing towards an improved understanding of the nearly universal behavior of this flow regime. Finally, they potentially open routes to turbulence control based on manipulation of nonlinear traveling waves.

\section{EXPERIMENTAL AND NUMERICAL SETUP}

The experiments were conducted in a rectangular duct with a width $(w)$ of $298 \mathrm{~mm}$, half-height (h) of $12.5 \mathrm{~mm}$, and length of $7.45 \mathrm{~m}$. The fluid used throughout this study was Newtonian, a 60:40\% by weight glycerine-water mixture. The flow was allowed to develop naturally, without the use of a turbulent trip, into a fully developed turbulent regime over the entire length of the duct, a distance of 596h. Wall-shear stresses were measured by a Dantec 55R46 flush-mounted miniaturized hot-film probe powered by a StreamLine Pro velocimetry system. The probe had a sensing element which was $0.2 \mathrm{~mm}\left(x^{+}=x u_{\tau} / v=1.38\right)$ long and $0.75 \mathrm{~mm}\left(z^{+}=5.19\right)$ wide and was flush mounted on the lower wall of the channel at $x / h=488, z / w=0.167$. Here, $x$ and $z$ denotes the streamwise and spanwise directions measured from inlet and the channel centerline, respectively; $u_{\tau}$ is the friction velocity at $R e_{\tau}=u_{\tau} h / v=85(R e=2400)$, and $v$ is the kinematic viscosity. The hot-film probe was calibrated in the channel flow facility by a Druck LPX-9381 low-differential pressure transducer. The pressure transducer estimated the streamwise pressure gradient, from which the mean wall shear stress could be determined, by measuring the difference in pressure across pressure taps installed on the lower wall of the stainless steel modules of the rectangular duct, over a distance of 2.05 $\mathrm{m}(x / h=164)$. The pressure transducer had a working range of $50 \mathrm{mbar}$, was accurate to $\pm 0.05 \mathrm{mbar}$, and was periodically calibrated against an MKS Baratron differential pressure transducer (1000 torr fsd). During the course of an experiment, the ambient fluid temperature would rise by typically $1^{\circ} \mathrm{C}$ 
due to the viscous heating of the pump. Since hot-film velocimetry is sensitive to temperature drift, the hot-film probe was calibrated before and after each experiment. The temperature during each experiment was carefully monitored by a platinum resistance thermometer (PRT) so that the voltage output from the hot-film probe could be linearly interpolated between the two calibration curves accordingly. The PRT was powered by an Agilent 34970A switch unit, which provided temperature readings with a resolution of $0.01{ }^{\circ} \mathrm{C}$ to an accuracy of $\pm 0.09^{\circ} \mathrm{C}$. Single-point measurements of the streamwise $(U)$ velocity were acquired at various wall-normal distances with a Dantec FibreFlow laser Doppler velocimetry (LDV) system, $12.5 \mathrm{~mm}$ downstream of the flush-mounted wall shear stress probe. The specially designed optical head on the LDV system provided a focal length of $160 \mathrm{~mm}$ and focused the laser beams to create a measurement volume with diameter of $0.025 \mathrm{~mm}\left(x^{+}=0.17\right)$ and length of $0.1 \mathrm{~mm}\left(z^{+}=0.69\right)$. Calibration experiments suggest that the LDV has an accuracy of about $1-2 \%$ in the mean velocity. The intersecting laser beams were traversed in the wall-normal direction through the channel flow via a custom-built traverse system, facilitating a spatial resolution of $1 \mu \mathrm{m}$ with accuracy of $3 \mu \mathrm{m}$. In a separate set of experiments, all three velocity components were measured simultaneously in the spanwise-wall-normal plane of the flow with a high-speed stereoscopic particle image velocimetry (SPIV) system, which provides global velocity measurements to an accuracy of typically 3-5\% [35]. The SPIV measurements were taken directly above the location of the flush-mounted wall shear stress probe. The numerical simulations were performed in an extended domain using the DNS code CHANNELFLOW [36], which solves the Navier-Stokes equations in the plane Poiseuille geometry using a Fourier-Chebyshev spatial discretization [37].

\section{RESULTS AND DISCUSSION}

To experimentally detect low-drag events we monitor the instantaneous wall shear stress at a point on the channel wall with a hot-film probe while simultaneously measuring the streamwise velocity at discrete distances above the wall with LDV or the whole flow field using SPIV. Shown in Fig. 1(a) are conditionally sampled low instantaneous wall shear stress events (thin gray lines) measured at $R e_{\tau}=100$. The laminar equivalent Reynolds number is $R e=U_{c} h / v$ and $R e_{\tau}=\sqrt{2 R e}$ (where $U_{c}$ is the laminar centerline velocity for the same pressure drop). Curves are shifted so that the instant when the wall shear stress falls $10 \%$ below the mean (dashed black line) corresponds to $t^{*}=t u_{\tau} / h=0$. Also shown (black line) is the ensemble average over all the instantaneous low wall shear stress events, and examples of instantaneous low-drag events with a duration $t_{d}^{*} \approx 4$ (blue line) and $t_{d}^{*} \approx 5$ (red line). Our selected criteria for hibernation was that the low stress event must last for a certain minimum duration (data shown here for $t_{d}^{*}>3$ but selecting $t_{d}^{*}>2.5$ or 3.5 gives essentially identical results). The distribution of the low stress events as a function of $t_{d}^{*}$ is shown in Fig. 1(b). Here, events with duration $t_{d}^{*}>5$ are detected, albeit infrequently, and are a function of Reynolds number. On average the hibernating wall shear stress falls to a plateau in the interval $t^{*}=0.7-2.8$ and is preceded for $-0.8<t^{*}<0$ by a brief interval of higher than average wall shear stress. These characteristics are observed at a range of Reynolds number and excellent qualitative agreement is found between the experiments and DNS in a large flow domain. Figure 1(c) shows that the DNS captures the essential features observed experimentally but slightly underpredicts the decrease in wall shear stress during a hibernating event. We attribute this small discrepancy to the inability to capture with DNS the boundary conditions and very large domain size of the experiment.

A universal feature of near-wall turbulent flows is a logarithmic dependence of the mean streamwise velocity $\left(U^{+}=U / u_{\tau}\right)$ with increasing distance from the wall $\left(y^{+}=y u_{\tau} / \nu\right)$. In Newtonian fluid flow, the mean streamwise velocity collapses to the von Kármán log law. In highly drag-reducing polymer solution flows, the mean streamwise velocity collapses to the so-called MDR asymptote $[24,33]$. In Fig. 2 we illustrate that the velocity field during these hibernating events in Newtonian turbulent channel flow for $70<R e_{\tau}<100$ essentially collapses to an MDR-like state that has hitherto been recognised experimentally only upon addition of drag-reducing additives to the flow. Open symbols in Fig. 2 are the usual ensemble-averaged streamwise velocity values, which fall onto the von Kármán log law (blue dotted line) once a sufficiently high Reynolds number is 

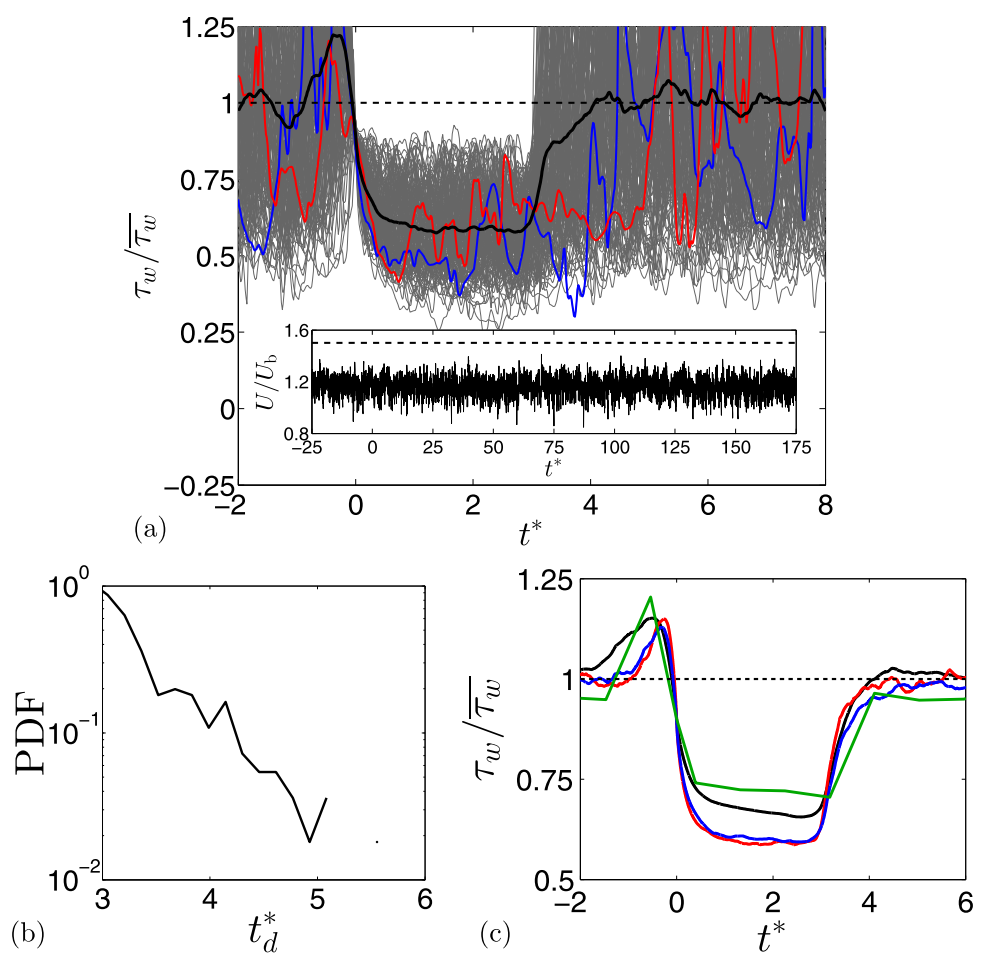

FIG. 1. (a) Example of instantaneous (thin gray lines) and ensemble-averaged (thick black line) experimental wall shear stress during intervals of hibernating turbulence at $R e_{\tau}=100(R e=5000)$. The thick blue and red lines highlight instantaneous low-drag events with durations of $t_{d}^{*} \approx 4$ and $t_{d}^{*} \approx 5$, respectively. The mean wall shear stress is shown by the black dashed line. The inset in panel (a) shows the instantaneous channel centerline velocity at $R e_{\tau}=100(R e=5000)$ with a hibernating turbulence interval beginning at $t^{*}=0$. The black dashed line shows the equivalent laminar centerline velocity, indicating that the flow does not relaminarize; this time series is representative for all Reynolds numbers studied. (b) The distribution of experimental low wall shear stress events as a function of $t_{d}^{*}$ at $R e_{\tau}=100(R e=5000)$ and (c) the ensemble-averaged wall shear stress during hibernating turbulence at $R e_{\tau}=70(R e=2450)$ (black line), $R e_{\tau}=85$ ( $\left.R e=3600\right)$ (blue line), $R e_{\tau}=100(R e=5000)$ (red line), and a DNS at $R e_{\tau}=85(R e=3600)$ (green line).

achieved, and are entirely consistent, even for higher-order turbulence statistics (not shown), with previous experimental results at higher $R e$ [38]. The closed symbols show the ensemble-averaged streamwise velocity measured during intervals of hibernation, averaged over the plateau region $t^{*}=0.7-2.8$ and scaled with the ensemble-averaged wall shear stress determined during the same time interval for LDV and the instantaneous wall shear stress for SPIV. These experimental data collapse very well to an MDR-like asymptote (in fact well within the uncertainty in the original data analysis that led to the MDR asymptote [39]: See the red dashed curve, indicating the lower end of the $95 \%$ confidence interval to the MDR asymptote. This curve is more representative of data at low $y^{+}$than is the canonical MDR log law shown as the solid red curve [33,39]); the uncertainty bars for the LDV data represent the spread of streamwise velocity data within each conditionally sampled ensemble average. Excellent agreement is also observed between the LDV and SPIV data sets. The conditionally sampled DNS velocity profile at $R e_{\tau}=85(R e=3600)$ is in good agreement with the experimental data until $y^{+} \approx 30$ after which it drops off slightly earlier from the MDR slope. Hence our low-Re data supports the hypothesis, certainly in terms of mean values, that the MDR and classical log laws represent an envelope of realizable dynamical turbulent states.

We now compare our results with the above-mentioned P4 ECS family in the minimal channel flow geometry [23], whose features are closely connected to the observations described above. 


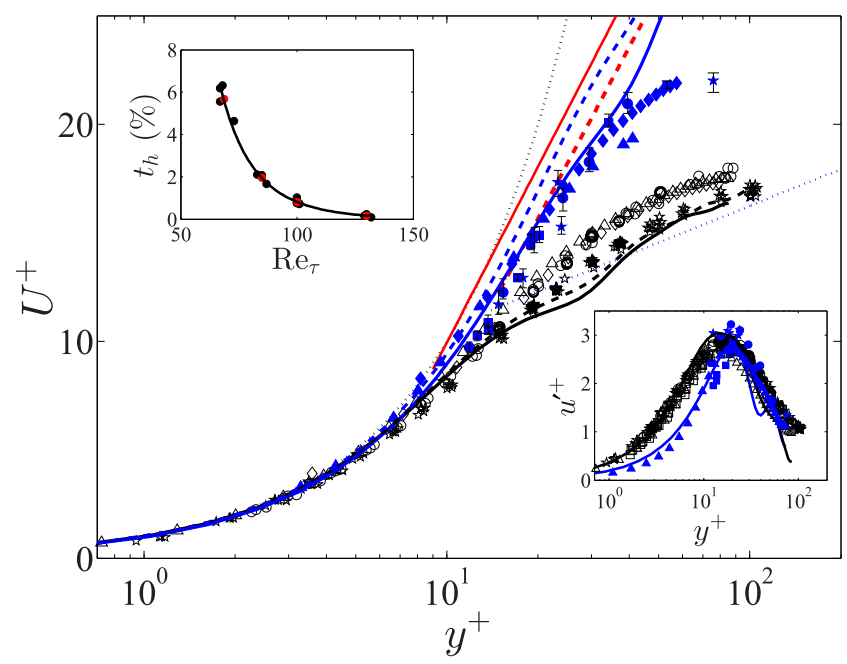

FIG. 2. Mean streamwise velocity scaled with mean wall shear stress (open symbols) and conditionally sampled ensemble-averaged streamwise velocity (hibernating turbulence data - closed symbols) scaled with conditionally sampled ensemble-averaged $\left(0.7<t^{*}<2.8\right)$ hibernating (low) wall shear stress: $R e_{\tau}=70(R e=$ 2450) ( $\square, \square), R e_{\tau}=85(R e=3600)(O, \bullet), R e_{\tau}=100(R e=5000)\left(\right.$ 约, $\star$ ), DNS at $R e_{\tau}=85(R e=3600)$ $(\triangle, \Delta)$, and SPIV at $R e_{\tau}=85(R e=3600)(\diamond, \diamond)$. Nonlinear TW solutions from Ref. [23] at $R e_{\tau}=85$ $(R e=3600)$ : lower branch (blue solid line) and upper branch (black solid line), and at $R e_{\tau}=100(R e=5000)$ : lower branch (blue dashed line) and upper branch (black dashed line). The black dotted line is the viscous sublayer profile: $U^{+}=y^{+}$, blue dotted line is the von Kármán log law $U^{+}=2.44 \ln y^{+}+5$, and the red solid line is canonical MDR log law: $U^{+}=11.7 \ln y^{+}-17$. The red dashed line shows the lower $95 \%$ confidence interval to the MDR asymptote [33,39]. The lower right inset shows streamwise velocity fluctuations $u^{\prime+}$. Symbols are as in the main figure. The upper left inset shows the proportion of time spent in hibernation $\left(t_{h}\right)$ vs $R e_{\tau}$ : individual experimental runs (๑), average number of events at $R e_{\tau}=70,85,100$, and $130(\bullet)$ and a (black) line to guide the eye.

Figure 2 includes average streamwise velocity profiles (with averaging over the full spatial periods in $x$ and $z$ ) for the high-drag and low-drag branches of this family at both $R e_{\tau}=85(R e=3600)$ and $100(R e=5000)$. The higher-drag (lower bulk velocity) branches, in black, called upper-branch solutions, have a bulk velocity that closely approaches that of active turbulence and the classical von Kármán log law, while the lower-branch solutions in blue bound the hibernation data and closely approach the Virk MDR profile.

The lower right inset in Fig. 2 shows $u^{\prime+}$ vs $y^{+}$for the experimental and DNS results above the measurement point (i.e., at $z^{+}=0$ ) along with results for the TWs. Individual hibernating events correspond to low-speed streaks that pass near the measurement point-thus the averaging that we do above the measurement point is essentially averaging over the width of a low-speed streak. Hence, for comparison, fluctuations for the lower branch TW are averaged in $z^{+}$over the low-speed streak region $-30<z^{+}<30$. The lower branch and the low-drag conditional results agree very closely, and likewise for the upper branch results and the unconditional averages. Thus the agreement between the experimental, DNS, and traveling wave results extends beyond the mean profiles.

Even at the transitonal Reynolds numbers studied here, turbulent channel flow enters states of hibernation only on infrequent occasions, with the detection of hundreds of hibernation events requiring several hours of sampling time. At the highest $R e$ studied $\left(R e_{\tau}=130\right)$, the number of hibernation events, although small, remains finite. The upper left inset in Fig. 2 shows the proportion of time spent in hibernation $\left(t_{h}\right)$ vs Reynolds number. Related observations of intermittent events with low and high turbulence activity have also been made in DNS of transitional pipe flow [40]. Indeed, the present results suggest that the quiescent regions arising during laminar-turbulent intermittency 

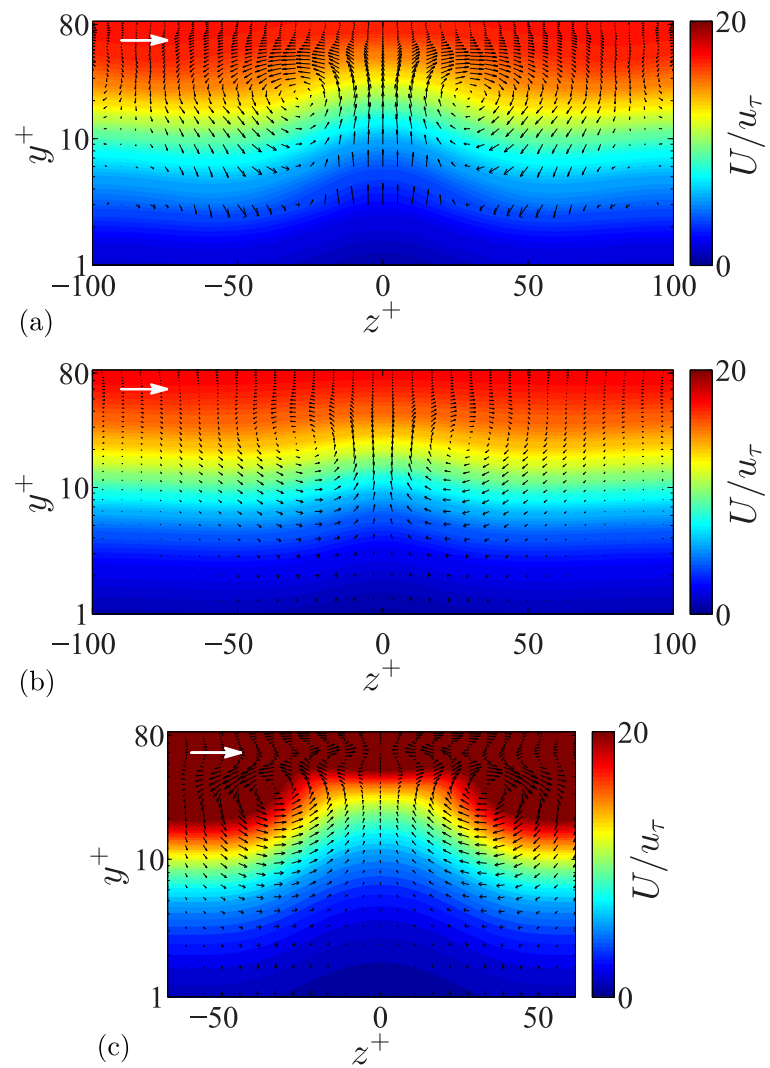

FIG. 3. Panels (a) and (b): Ensemble-averaged streamwise velocity, normalized by the time-averaged friction velocity $\left(u_{\tau}\right)$ at $R e_{\tau}=85(R e=3600)$ during hibernating turbulence events (a) captured using SPIV and (b) from DNS. The ensemble-average comprises 207 PIV snapshots acquired over four low-drag events in (a) and 1302 DNS snapshots over 651 low-drag events in panel (b). The correlation coefficient between the SPIV and DNS velocity magnitudes is 0.995 . As part of the ensemble averaging the data are symmetrized about $z^{+}=0$, which is the detection point of the hibernating turbulence intervals. (c) Streamwise-averaged streamwise velocity for the lower branch TW solution at $R e_{\tau}=85(R e=3600)$. The color bar maximum value is limited to highlight near-wall dynamics (for the TW solution the centerline velocity is $\approx 34 u_{\tau}$ ). Black arrows show the averaged wall-normal and spanwise velocities. The white arrows have length $0.5 u_{\tau}$.

are spatially local approaches to lower branch traveling waves, at least in some parts of parameter space. This idea is consistent with low Re minimal channel DNS results, which show temporal intermittency between upper and lower branch states [23].

Although these forays into hibernation with a Newtonian fluid are rare, previous numerical simulations suggest that the addition of viscoelasticity from a polymer additive suppresses the dominant turbulent motions and entices the flow to enter a state of hibernation more frequently $[28,31]$. On a time-averaged point of view, this yields a large reduction in turbulent skin-friction drag, and causes the polymer solution's streamwise velocity profile to approach the MDR asymptote. As the level of viscoelasticity increases, it has a stabilizing or reinforcing effect on the dynamics, leading to important changes in the turbulent fluctuations at MDR [33,34,41].

Finally, Figs. 3(a)-3(c) show conditional-sampled ensemble-averaged flow structures during hibernation identified from experiments, DNS, and a lower branch ECS solution at $R e_{\tau}=85$, respectively (see time series incorporating hibernation intervals in Supplemental Movies 1, 2, and 3 [42]). The ensemble averages include snapshots over the whole duration of the low-drag event, 
i.e., at different points in time as the structure is moving through the measurement plane. In other words, in the experiments and DNS there is an effective streamwise averaging so it is appropriate to do the same with the traveling waves. Color indicates streamwise velocity while arrows denote velocity in the $z-y$ plane. We see that hibernating turbulence is characterized by a counter-rotating streamwise vortex pair, which induces a low-speed and low-stress streak in the near-wall region of the flow. Far from the wall the TW [Fig. 3(c)] remains coherent with only a very weak structure, while the turbulent flows are less so, and thus have lower mean velocity. The vortex-pair structures found in all three cases are in very good agreement, with very similar magnitudes for all velocity components but slightly different positions for the streamwise vortex cores $\left[\left(z^{+}, y^{+}\right) \approx(34,33)\right.$ for the TW and $(29,24)$ for both DNS and experiments].

\section{CONCLUSIONS}

To conclude, we recapitulate the main results in context with other recent observations. In minimal channels at transitional Reynolds number, turbulent trajectories display intermittent low-drag events in which the turbulent trajectory moves toward the lower branch P4 exact coherent state identified in Ref. [23]. This state has a mean velocity profile that is close to the MDR velocity profile observed for polymer solutions [24]. The present work characterizes low-drag events in extended domains, also at low $R e$, using both experiments and computations, showing that in terms of mean profiles, streamwise fluctuations, and structure of the streamwise velocity fluctuations, these events also display close similarities in the region $y^{+} \lesssim 30$ with the P4 ECS found in the minimal domain. These similarities suggest that there is a significant connection that is not yet understood between temporal intermittency in minimal domains and spatiotemporal intermittency in extended domains, and that this connection is mediated by underlying ECS in the state space of the Navier-Stokes equations. Furthermore, the similarity of the mean velocity profile during the low-drag events to the MDR profile in polymer solutions is consistent with past work $[29,32]$ in minimal channels that at low to moderate drag reduction levels the main effect of polymers is to increase the frequency of low-drag events while leaving their structure virtually unchanged. These observations form a basis for future work in understanding laminar-turbulence intermittency, drag reduction by polymers, and possibly in flow control for drag reduction.

\section{ACKNOWLEDGMENTS}

This work has been supported by the Engineering and Physical Sciences Research Council (EPSRC) under Grant No. EP/J018163/1, the National Science Foundation through Grant No. CBET-1066223, and the Air Force Office of Scientific Research through Grants No. FA9550-11-10094 and No. FA9550-15-1-0062 (Flow Interactions and Control Program). The direct numerical simulation code used here was developed and distributed by John Gibson at the University of New Hampshire. We thank the EPSRC for the loan of the SPIV system used to carry out this research.

[1] S. K. Robinson, Coherent motions in the turbulent boundary layer, Annu. Rev. Fluid Mech. 23, 601 (1991).

[2] J. Jiménez and A. Pinelli, The autonomous cycle of near-wall turbulence, J. Fluid Mech. 389, 335 (1999).

[3] J. M. Hamilton, J. Kim, and F. Waleffe, Regeneration mechanisms of near-wall turbulence structures, J. Fluid Mech. 287, 317 (1995).

[4] F. Waleffe, On a self-sustaining process in shear flows, Phys. Fluids 9, 883 (1997).

[5] W. Schoppa and F. Hussain, Coherent structure generation in near-wall turbulence, J. Fluid Mech. 453, 57 (2002).

[6] G. Kawahara, M. Uhlmann, and L. van Veen, The significance of simple invariant solutions in turbulent flows, Annu. Rev. Fluid Mech. 44, 203 (2012). 
[7] B. Hof, C. W. H. van Doorne, J. Westerweel, F. T. M. Nieuwstadt, H. Faisst, B. Eckhardt, H. Wedin, R. R. Kerswell, and F. Waleffe, Experimental observation of nonlinear traveling waves in turbulent pipe flow, Science 305, 1594 (2004).

[8] F. Waleffe, Exact coherent structures in channel flow, J. Fluid Mech. 435, 93 (2001).

[9] M. Nagata, Three-dimensional finite-amplitude solutions in plane Couette flow: Bifurcation from infinity, J. Fluid Mech. 217, 519 (1990).

[10] R. M. Clever and F. H. Busse, Tertiary and quaternary solutions for plane Couette flow, J. Fluid Mech. 344, 137 (1997).

[11] M. Nagata, Three-dimensional traveling-wave solutions in plane Couette flow, Phys. Rev. E 55, 2023 (1997).

[12] F. Waleffe, Three-Dimensional Coherent States in Plane Shear Flows, Phys. Rev. Lett. 81, 4140 (1998).

[13] F. Waleffe, Homotopy of exact coherent structures in plane shear flows, Phys. Fluids 15, 1517 (2003).

[14] H. Faisst and B. Eckhardt, Traveling Waves in Pipe Flow, Phys. Rev. Lett. 91, 224502 (2003).

[15] H. Wedin and R. R. Kerswell, Exact coherent structures in pipe flow: Traveling wave solutions, J. Fluid Mech. 508, 333 (2004).

[16] J. F. Gibson, J. Halcrow, and P. Cvitanovic, Visualizing the geometry of state space in plane Couette flow, J. Fluid Mech. 611, 107 (2008).

[17] J. F. Gibson, J. Halcrow, and P. Cvitanovic, Equilibrium and traveling-wave solutions of plane Couette flow, J. Fluid Mech. 638, 243 (2009).

[18] T. M. Schneider, J. F. Gibson, and J. Burke, Snakes and Ladders: Localized Solutions of Plane Couette Flow, Phys. Rev. Lett. 104, 104501 (2010).

[19] A. de Lozar, F. Mellibovsky, M. Avila, and B. Hof, Edge State in Pipe Flow Experiments, Phys. Rev. Lett. 108, 214502 (2012).

[20] T. Kreilos, G. Veble, T. M. Schneider, and B. Eckhardt, Edge states for the turbulence transition in the asymptotic suction boundary layer, J. Fluid Mech. 726, 100 (2013).

[21] H. M. Blackburn, P. Hall, and S. J. Sherwin, Lower branch equilibria in Couette flow: The emergence of canonical states for arbitrary shear flows, J. Fluid Mech. 726, R2 (2013).

[22] J. Jimenez and P. Moin, The minimal flow unit in near-wall turbulence, J. Fluid Mech. 225, 213 (1991).

[23] J. S. Park and M. D. Graham, Exact coherent states and connections to turbulent dynamics in minimal channel flow, J. Fluid Mech. 782, 430 (2015).

[24] P. S. Virk, Drag reduction fundamentals, AIChE J. 21, 625 (1975).

[25] C. F. Li, V. K. Gupta, R. Sureshkumar, and B. Khomami, Turbulent channel flow of dilute polymeric solutions: Drag reduction scaling and an eddy viscosity model, J. Non-Newton. Fluid Mech. 139, 177 (2006).

[26] R. R. Kerswell, D. Obrist, and P. J. Schmid, On smoothed turbulent shear flows: Bounds, numerics and stress-reducing additives, Phys. Fluids 15, 78 (2003).

[27] P. R. Bandyopadhyay, Stokes mechanism of drag reduction, J. Appl. Mech. 73, 483 (2006).

[28] L. Xi and M. D. Graham, Turbulent drag reduction and multistage transitions in viscoelastic minimal flow units, J. Fluid Mech. 647, 421 (2010).

[29] L. Xi and M. D. Graham, Active and Hibernating Turbulence in Minimal Channel Flow of Newtonian and Polymeric Fluids, Phys. Rev. Lett. 104, 218301 (2010).

[30] Y. Dubief, C. M. White, E. S. G. Shaqfeh, and V. E. Terrapon, Polymer maximum drag reduction: A unique transitional state, in Annual Research Briefs (Center for Turbulence Research, Stanford, CA, 2010), pp. 395-404.

[31] L. Xi and M. D. Graham, Dynamics on the Laminar-Turbulent Boundary and the Origin of the Maximum Drag Reduction Asymptote, Phys. Rev. Lett. 108, 028301 (2012).

[32] L. Xi and M. D. Graham, Intermittent dynamics of turbulence hibernation in Newtonian and viscoelastic minimal channel flows, J. Fluid Mech. 693, 433 (2012).

[33] M. D. Graham, Drag reduction and the dynamics of turbulence in simple and complex fluids, Phys. Fluids 26, 101301 (2014).

[34] S. N. Wang, M. D. Graham, F. J. Hahn, and L. Xi, Time-series and extended Karhunen-Loève analysis of turbulent drag reduction in polymer solutions, AIChE J. 60, 1460 (2014). 
[35] J. Westerweel, Fundamentals of digital particle image velocimetry, Meas. Sci. Technol. 8, 1379 (1997).

[36] J. F. Gibson, Channelflow: A spectral Navier-Stokes simulator in C++, Tech. Rep., University of New Hampshire, Durham, 2014 (unpublished).

[37] For the DNS results, the computational domain is $L_{x} \times 2 h \times L_{z}$ with $L_{x}=35.36 h$ and $L_{z}=9.43 h$ being the length and width of the domain in the $x$ (streamwise) and $z$ (spanwise) directions, respectively. At $R e_{\tau} \approx 85$, this corresponds to a domain size of approximately $3000 \times 800$ in inner units, or about $5 \times 8$ correlation lengths. A numerical grid system is generated on $160 \times 73 \times 120$ collocation points in $x, y$, and $z$, respectively.

[38] T. Wei and W. W. Willmarth, Reynolds-number effects on the structure of a turbulent channel flow, J. Fluid Mech. 204, 57 (1989).

[39] P. S. Virk, H. S. Mickley, and K. A. Smith, The ultimate asymptote and mean flow structure in Toms' phenomenon, J. Appl. Mech. 37, 488 (1970).

[40] M. Avila and B. Hof, Nature of laminar-turbulence intermittency in shear flows, Phys. Rev. E 87, 063012 (2013).

[41] D. Samanta, Y. Dubief, M. Holzner, C. Schäfer, A. N. Morozov, C. Wagner, and B. Hof, Elasto-inertial turbulence, Proc. Natl. Acad. Sci. USA 110, 10557 (2013).

[42] See Supplemental Material at http://link.aps.org/supplemental/10.1103/PhysRevFluids.2.034602 for Supplementary Movie 1 (experiment) and Movie 2 (DNS) show the temporal evolution of turbulent flow structure and wall shear stress during a single hibernating turbulence event in a fully developed turbulent channel flow at $R e_{\tau}=85(R e=3600)$. The streamwise velocity is normalized by the channel centreline velocity $\left(U_{C L}\right)$, and the white arrow in the top left hand corner is a reference vector showing $0.5 U_{C L}$. Supplementary Movie 3 shows the temporal evolution of flow structure for the lower branch of the traveling-wave solution during one period at $R e_{\tau}=85(R e=3600)$. The structure is moving at constant speed in the positive $x^{+}$direction. The streamwise velocity is represented by color contours with blue indicating low speed and red indicating high speed. The wall-normal and spanwise velocities are shown by arrows, which are on the same scale for both movies. Dark red tubes are isosurfaces of constant swirling strength and illustrate slightly inclined vortical flow structures. Transparent blue isosurfaces indicate critical layer surfaces, where the local streamwise velocity matches the wave speed. 